\title{
Accounting for species' thermodynamic activities changes mechanistic interpretations of electrochemical kinetic data
}

\author{
Kindle Williams, ${ }^{\mathrm{a}}$ Aditya Limaye, ${ }^{\mathrm{a}}$ Trent Weiss, ${ }^{\mathrm{a}}$ Minju Chung, ${ }^{\mathrm{a}}$ Karthish Manthiram ${ }^{\mathrm{a}, *}$ \\ ${ }^{a}$ Department of Chemical Engineering, Massachusetts Institute of Technology, Cambridge, MA \\ 02139, United States
}

* Corresponding author. Email: karthish@mit.edu

\begin{abstract}
The thermodynamic activity of a reacting species, rather than the concentration of that species, generally determines the rate of a kinetically-limited reaction. In this work we demonstrate the need for the explicit accounting of reacting species' thermodynamic activities in solution, especially when conducting electrochemical kinetic tests. In hydrogen evolution in an alkaline acetonitrile-water blended electrolyte as well as previously-reported oxygen-atom transfer reactions (cyclooctene epoxidation and cyclohexanone lactonization), we demonstrate that accounting for species thermodynamic activity causes water-dependence measurements to yield different mechanistic interpretations than data which treats concentration as a proxy for activity. We hypothesize many ways in which water contributes to the reaction rate beyond direct participation in the reaction, offer initial molecular interpretations of the water activityconcentration relationship in the blended electrolyte, and discuss implications of these findings for better understanding solvent effects.
\end{abstract}

\section{Introduction}

Electrochemical reactions are some of the many tools at society's disposal for aiding in renewable energy storage, decarbonization of the chemical industry, modular/low-temperature and -pressure transformations, and selective chemical synthesis. ${ }^{1}$ In the study of these reactions, attention has been paid to the design of both the electrode/catalyst material used to facilitate charge transfer as well as the electrolyte - that is, the medium through which ionic conduction occurs between the anode and the cathode. In engineering liquid/solvated electrolytes, electrochemists are increasingly interested in solvent ${ }^{2-5}$ and electrolyte salt ${ }^{6-8}$ effects on reaction rates and mechanisms. Recent work has also demonstrated the usefulness of operating in mixed-solvent environments. For example, some $\mathrm{O}$-atom transfer (OAT) reactions require contacting water - the $\mathrm{O}$-atom source with organic substrates, which are often relatively water-insoluble hydrocarbons. ${ }^{9,10}$ An organic solvent is often used to bring these species into contact with one another in a single phase as an alternative to approaches involving two-phase ${ }^{11-13}$ or segmented flow ${ }^{14}$ setups. Acetonitrile is an example of such a solvent, often chosen due to its relative oxidative stability in the absence of a 
co-solvent. ${ }^{15}$ The resulting electrolyte, consisting of organic solvent, water, salt, and any substrates of interest, we will here refer to as a "blended electrolyte" (or, in the absence of electrolyte salt, simply "blended solvent"). These electrolytes exhibit interesting intermolecular interactions and properties at the nanoscale, such as the clustering of water into so-called "nanoreactors.",

The literature is rich with investigations of the structure and properties of blended solvent systems outside the contexts of electrochemistry and catalysis. For instance, the proton-solvation properties of solutions consisting of water and amines, ${ }^{16}$ nitriles, and alcohols ${ }^{17}$ have been investigated. Techniques such as infrared (IR) spectroscopy, ${ }^{18}$ nuclear magnetic resonance (NMR), ${ }^{19}$ X-ray absorption spectroscopy (XAS), ${ }^{20,21}$ and molecular dynamics (MD) simulation ${ }^{19}$ have been applied to study the structure and bonding of components in blended solvents. Recently, much attention has been directed toward understanding blended electrolytes consisting of water and charged components such as ionic liquids, ${ }^{22}$ as well as toward high-concentration salts in so-called "waterin-salt" electrolytes. ${ }^{23}$ However, relatively little work in catalysis has been directed toward quantifying the impact of these structural changes brought about due to nonideal solvent mixtures - that is, solvent mixtures in which the activity coefficient of species of interest is not constant across the concentration range of interest (see The relevance of species activities in rate laws).

Blended solvents have been employed in catalysis to varying extents based on application. In homogeneous catalysis, water/organic blended solvents have been used to illustrate solvent effects for acid-catalyzed biomass conversion. ${ }^{24-26}$ Homogeneous hydrogen evolution reports utilizing transition metal complex catalysts have also implemented blended electrolytes consisting of organic solvents alongside organic acids acting as proton donors. ${ }^{27,28}$ In general, research pertaining to hydrogen evolution (HER) and oxidation (HOR) has paid the most explicit attention to the impact of water content and structure on heterogeneous catalysis, both in purely aqueous electrolytes, where interfacial water structure has become increasingly emphasized, ${ }^{29-40}$ and in blended electrolytes. ${ }^{7,41-43}$ Recent interest in decoupling the role of water as a proton source and as a solvent in heterogeneous HER and HOR has prompted novel investigations of the reactions in water/acetonitrile mixtures ${ }^{32,41,42,44}$ as well as in water/carbonate-based electrolytes. ${ }^{45}$ These works have utilized limited approaches to perform fundamental kinetic characterization on the reactions at hand that could decouple the many roles of water in these reactions; however, questions of thermodynamics with respect to potential scales and proper referencing techniques are increasingly being addressed. ${ }^{46,47}$

Outside of HER and HOR, electrochemical applications of blended electrolytes have focused less on the specific roles of water in reactions. Instead, reports have emphasized the impact of blended electrolyte composition on redox potentials for homogeneous complexes ${ }^{48}$ redox potentials for the various carbon dioxide reduction reactions $\left(\mathrm{CO}_{2} \mathrm{RR}\right),{ }^{49}$ and effects of added acetonitrile in blended electrolytes for oxygen reduction $(\mathrm{ORR})^{50}$ and formic acid oxidation. ${ }^{51} \mathrm{In}$ addition, research efforts utilizing water as an O-atom source have also historically been conducted in blended electrolytes. While some of these works included explicit measurements of the dependence of reaction rate on water concentration, ${ }^{9,10,52}$ no extensive treatment has been given in any of these works to the nonidealities of the electrolyte mixture.

In this work, we directly account for the nonideal thermodynamics of water in a blended electrolyte, and use this accounting to better understand measured dependences of heterogeneous electrocatalytic reaction rates on water. We conduct novel investigations on the water dependence of alkaline HER in acetonitrile, and also look back at previously-reported water dependences for 
electrocatalytic O-atom transfer chemistries. In addition, we discuss the structure and energetics of water in a simulated blended electrolyte, and offer a number of explanations for the many roles water plays in blended electrolyte electrocatalysis. While water is an interesting edge case when it comes to intermolecular interactions, many of these roles also pertain to nonaqueous co-solvents, lending to the broad applicability of these conclusions.

\section{Results}

The relevance of species activities in rate laws

When studying electrochemical reactions, one useful goal might be to achieve mechanistic understanding of a reaction, often with an associated reaction rate expression. For an arbitrary reaction, a generic rate law can be written from Transition State Theory:

$$
r=\frac{k}{\gamma_{\ddagger}} \prod_{j} a_{j}^{v_{j}}
$$

where $r$ is the reaction rate, $k$ is a reaction rate constant which subsumes details about temperature as well as energetics of the transition state, $\gamma \neq$ is the activity coefficient of the transition state, and $a_{j}$ represents the activity of species $j$ with corresponding stoichiometric coefficient $v_{j}$ (see Supplementary Information for a full treatment of this rate formulation). That reaction rate laws should be formulated using species activities rather than species concentrations is well-established within the heterogeneous catalysis community, where explicit tracking of transition state nonidealities has been a subject of discussion. ${ }^{53}$ The relationship between activity and concentration can be written as:

$$
a_{j}=\frac{\gamma_{j} c_{j}}{c_{j, 0}}
$$

where $\gamma_{j}$ is the activity coefficient of species $j, c_{j}$ is the concentration of species $j$, and $c_{j, 0}$ is a reference concentration at which activity is typically set to be unity. Many researchers tend to apply the simplifying assumption that the activity coefficients $\gamma_{j}$ are constant for a given species at all tested conditions - in other words, the reactants are assumed to behave ideally in solution. To assess the validity of this assumption, we can examine the anatomy of a previously measured activity-concentration relationship for a binary mixture. ${ }^{54,55}$ (Figure 1) 


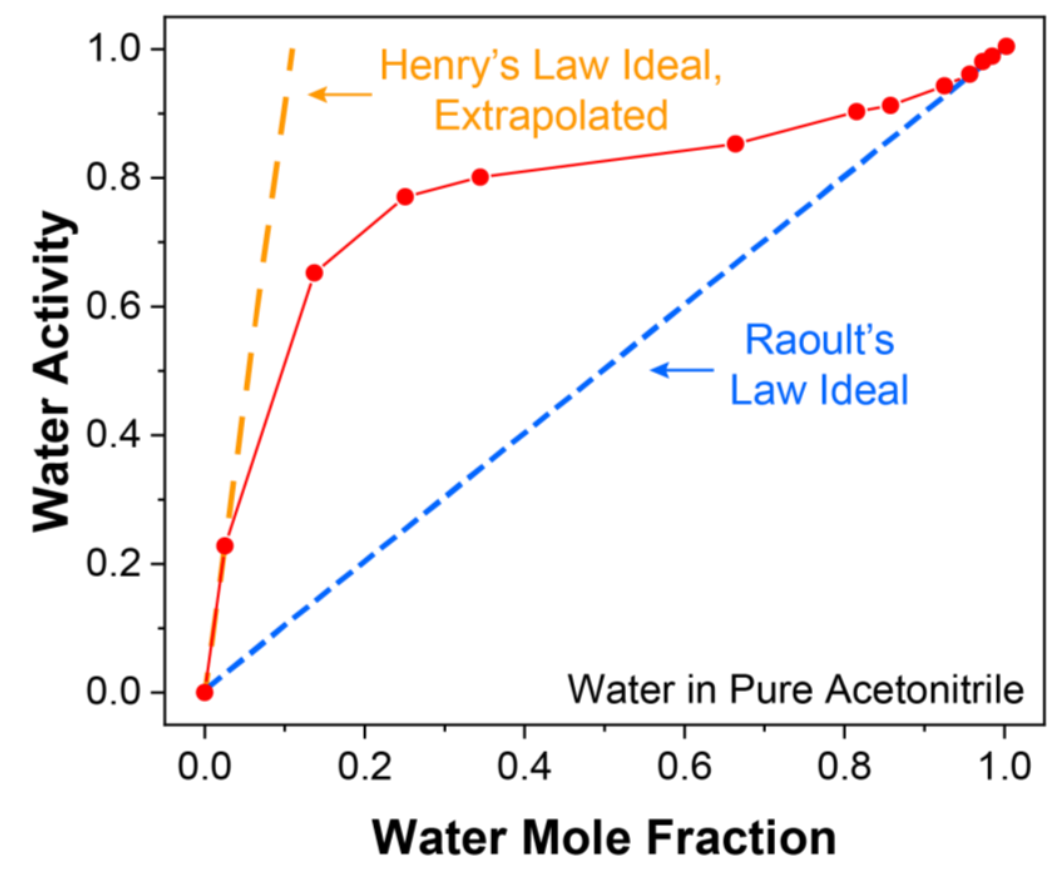

Figure 1. Illustrative concentration-activity relationship for a binary mixture of water and acetonitrile. The dashed orange and blue lines roughly indicate the Henry's Law (dilute water reference) and Raoult's Law (pure water reference) ideal cases, respectively. Data extracted from Sirotkin et al. .55 originally collected by Treiner et al. ${ }^{54}$

For this binary mixture of water and acetonitrile, we have noted the two distinct types of ideality using dashed lines. In the first type, the species of interest (here water) is assumed to be infinitely dilute in the solvent of interest, with each water molecule assumed not to interact to a great extent with other water molecules around it. In this formulation, we can refer to a species as being ideal with respect to Henry's law (orange dashed line). Here, concentration and activity can be related by:

$$
a_{j}=\frac{c_{j}}{H_{j}}
$$

where $H_{j}$ is the Henry's law constant for species $j$, and is the inverse slope of the dilute- $j$ limit of the activity-concentration relationship, equivalent to $H_{j}=c_{j, 0} / \gamma_{j}$. This formulation is often applied to dilute salts and dissolved gases in solution. On the other hand, the Raoult's law ideality formulation (blue dashed line) is one in which a saturated reference is used - a reference in which the species of interest is only engaging in self-interactions. For this formulation,

$$
a_{j}=\frac{c_{j}}{c_{j, \infty}}
$$

Where $c_{j, \infty}$ is a saturated reference state, equivalent to $x_{j}=1$. This would imply that for a Raoult's law ideal mixture, $\gamma_{j}=1$. 
Examining the activity-concentration relationship for water and acetonitrile, it can be observed that an ideal formulation of either variety is only applicable across a very narrow range of concentrations. In a blended aqueous-nonaqueous system, we must consider the middle region, in which the relationship exhibits curvature. For most fully-miscible binary mixtures, the relationship will take this general shape: first upward, deviating positively from Raoult's law, then with decreasing slope, then curving upward to rejoin Raoult's law at high $x_{j}$. The positive deviation from Raoult's law across the middle of the plot indicates that species $j$ is engaging in selfinteractions that are more favorable than interactions with the other component of the mixture. Note that in these cases $\gamma_{j}>1$. Water, being uniquely polar and strongly $\mathrm{H}$-bonding, tends to have a large preference for self-interactions. As a result, the downward curvature at intermediate water concentrations leads to a very obvious "plateau" in activity. We hypothesize this is due to clustering of water at the nanoscale, though it appears discussion of the molecular origins of activity coefficient have been sparse in the literature.

Thus, in the case in which water and acetonitrile are the primary components of a blended electrolyte, it is necessary to apply a correction to measured data in which the composition of the electrolyte is changing. It should be noted that this conclusion generally applies to any electrolyte or reaction solution in which activity coefficients might reasonably be changing throughout a given set of tests.

\section{Hydrogen evolution as a demonstration of the activity correction}

In order to clearly illustrate the importance of the activity correction in a nonideal electrolyte mixture, we studied the water dependence in a blended electrolyte of one of the simplest, most well-studied electrocatalytic reactions: the hydrogen evolution reaction (HER). HER represents the cathodic half-reaction in oxidative electro-organic transformations such as oxygen-atom transfer chemistries ${ }^{9,10}$ and is a foundational chemistry to understand in any solvent system under consideration for practical deployment. We elected to characterize HER on a Au rotating disk electrode (RDE) cathode using an electrolyte consisting of acetonitrile, water, $0.778 \mathrm{M} \mathrm{TBABF}_{4}$, and $0.022 \mathrm{M}$ tetrabutylammonium hydroxide (TBAOH). (Figure 2) Including base helped to ensure that water was the reactant rather than protons, and that there was no large $\mathrm{pH}$ swing in the working electrolyte throughout the course of the experiment. HER on Pt in this electrolyte was also studied, but was found to exhibit substantial shifts in Tafel slope at different values of water activity, suggesting (among other possibilities) an inconsistent reaction mechanism; those results may be found in the Supplementary Information. 

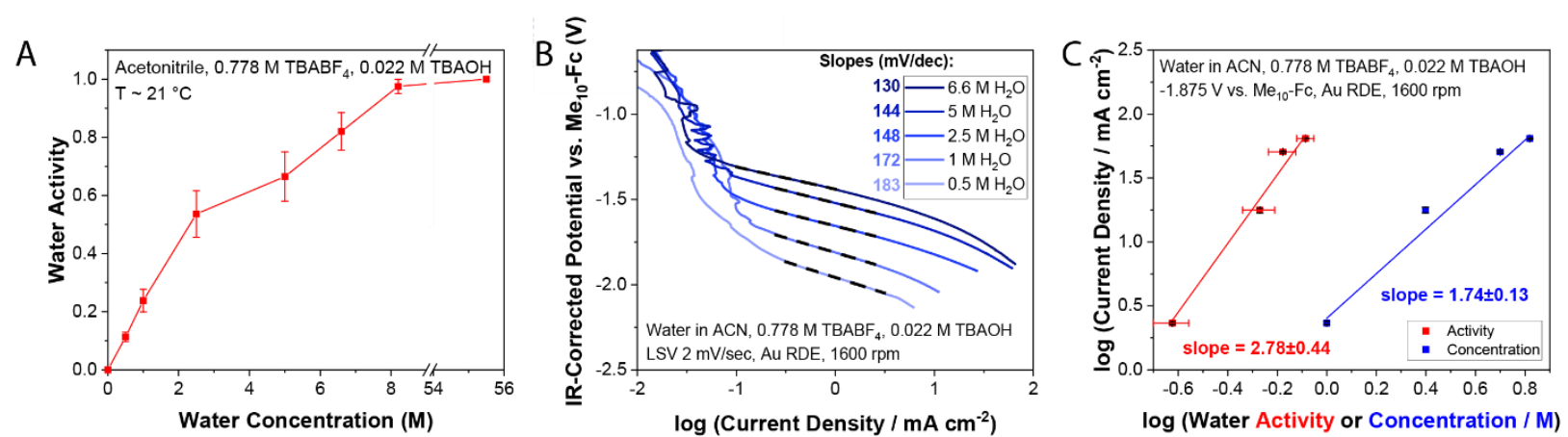

Figure 2. Data collected for the purpose of evaluating the mechanism of HER on Au in basic blended electrolyte. A) Concentration-activity relationship for water in acetonitrile with $0.778 \mathrm{M}$

$\mathrm{TBABF}_{4}, 0.022 \mathrm{M} \mathrm{TBAOH}$, measured using HS-GC-TCD. Error bars represent standard deviation from triplicate measurements. B) Tafel plots for HER at multiple concentrations of water in basic blended electrolyte (water in acetonitrile with $0.0778 \mathrm{M} \mathrm{TBABF}_{4}, 0.022 \mathrm{M}$ TBAOH), collected on Au RDE under Ar atmosphere (deoxygenated electrolyte) by conducting linear sweep voltammograms at a sweep rate of $2 \mathrm{mV} / \mathrm{sec}$ from $-0.5 \mathrm{~V}$ vs. Me $\mathrm{Me}_{10}-\mathrm{Fc}$ to roughly $2.0 \mathrm{~V}$ vs. $\mathrm{Me}_{10}-\mathrm{Fc}$. C) Water dependence of HER evaluated at a single potential, here $-1.875 \mathrm{~V}$ vs. $\mathrm{Me}_{10}$-Fc. X-error bars reflect errors in activity measurements, while y-error bars reflect measured error in Me10-Fc calibrations. Further treatment of errors can be found in the Supplementary Information.

Since the water dependence of HER in a blended electrolyte has never been reported, a new protocol was developed for this purpose. This protocol consisted of four main steps: 1) directly measuring water activity in the electrolytes of interest; 2) calibrating reference electrodes in those electrolytes; 3) performing linear sweep voltammograms in those electrolytes; and 4) combining all the data to yield a water dependence measurement for HER. Detailed experimental methods for each of these steps may be found in the Supplementary Information.

In order to measure water activity, vapor-liquid equilibrium was invoked, which is to say: $\mu$ liquid $=$ $\mu_{\text {vapor, }}$ where $\mu$ represents species chemical potential. A headspace gas sampling protocol was established in which the vapor that had equilibrated over each electrolyte mixture could be sampled and characterized. (see Methods) This protocol was used to generate Figure 2A. It may be noted that the high concentration of the tetrabutylammonium salt, which is relatively hydrophobic, served to elevate water activity relative to that in a binary water-acetonitrile mixture at a given water mole fraction. (Figure 1) This is because unfavorable interactions in general increase species activity. The hydrophobicity of the salt also caused visual phase separation at $c_{\mathrm{H} 2 \mathrm{O}}=8.3 \mathrm{M}$, at which concentration the measured water activity was found to be within error of unity.

It may be noted that the activity measurements described above are bulk measurements, whereas heterogeneous electrocatalysis is an interfacial phenomenon. Why then should a bulk measured species activity value be applicable to reactions taking place at a surface? The surface environment is clearly quite different from the bulk, in ways ranging from specific surface interactions to features of the interfacial electric field. ${ }^{56}$ The orientation and concentration of species in this region should also differ from that in the bulk. However, we emphasize that species activity measured in the bulk should be indicative of species activity at a surface in equilibrium with that bulk as long 
as there are no transport-induced limitations impeding the equilibrium between the bulk and surface, i.e. $\mu_{j, b u l k}=\mu_{j, \text { surf. }}$. Based on the definition of activity, this in turn means:

$$
a_{j, b u l k}=e^{\left(\mu_{j, b u l k}-\mu_{j, 0}\right) / R T}=e^{\left(\mu_{j, s u r f}-\mu_{j, 0}\right) / R T}=a_{j, s u r f}
$$

That is to say, regardless of any factors at an electrode surface that might affect species orientation and abundance - e.g., hydrophobicity, catalyst morphology, and polarization - bulk measured activity is equivalent to surface activity so long as surface fluxes are not so high that transport limitations cannot be neglected.

In order to establish a measure of electrochemical potential in each composition of the blended electrolyte, a leak-free $\mathrm{Ag} / \mathrm{AgCl}$ pseudoreference electrode was calibrated versus decamethylferrocene $\left(\mathrm{Me}_{10}-\mathrm{Fc}\right)$, an electron-transfer $(\mathrm{ET})$ reference species whose redox potential demonstrates a high degree of solvent-independence. ${ }^{57}$ It should be noted that the potential of an ET reference is distinct from a pH-dependent reference such as the reversible hydrogen electrode, RHE. Referencing against RHE would yield information about HER at a constant overpotential a value that accounts for the thermodynamics of the proton transfer. Both the potential of an ET reference such as $\mathrm{Me}_{10}-\mathrm{Fc}$ as well as that of a coupled proton-electron transfer (CPET) reference such as RHE can be measured in any arbitrary electrolyte. ${ }^{46,58,59}$ Both referencing schemes are informative, especially in the context of one another - the difference between results referenced to the ET versus CPET can be attributed specifically to the thermodynamics of the proton transfer. For the purposes of this discussion, we will primarily examine the water dependence of HER at a constant potential versus an ET reference rather than at constant overpotential; results referenced to RHE as well as a further discussion of insights offered by those results can be found in the Supplementary Information.

After obtaining the water activity-concentration relationship, as well as calibrating the reference electrode, HER experiments were conducted. Linear sweep voltammetry was performed using a polished, pre-oxidized Au disk in RDE configuration to generate Tafel plots (Figure 2B) as well as water dependence measurements (Figure 2C). (see Methods) It should be noted that while the RDE was spun at $1600 \mathrm{rpm}$ for these measurements, similar tests were also conducted at 900 and $2500 \mathrm{rpm}$ to demonstrate that transport limitations did not play a substantial role in the measurements (see Supplementary Information).

From the measured Tafel slopes of $130-183 \mathrm{mV} / \mathrm{dec}$, we can conclude that the mechanism of HER on $\mathrm{Au}$ in the blended electrolyte media likely involves a rate-determining step with a cardinal Tafel slope value of $118 \mathrm{mV} / \mathrm{dec}$, such as the Volmer step (initial electron transfer to a proton donor), the Heyrovsky step at high surface coverage (electron transfer to a solution-phase proton donor following adsorption of $\mathrm{H}^{*}$ ), or surface diffusion at moderate surface coverage (see Supplementary Information). ${ }^{60,61}$ Of these, the Volmer is the most likely rate-determining step, as this aligns with previous reports on aqueous alkaline HER on Au. ${ }^{62}$ The measured Tafel slope decreases with increasing water content - or in other words, an increased presence of water leads to a larger current response to a given change in potential. It is possible that the difference in slopes is in some way related to the fact that the Tafel fits are performed at different potential ranges (see below for more details). Note that while the reported Tafel slopes were not fit using a Bayesian approach ${ }^{63}$ 
due to persistent ORR background (see Supplementary Information), sensitivities to the fit range are reported in the Supplementary Information.

With the assertion that the Volmer step is rate-limiting comes the physical picture of a single water molecule donating a proton which combines with an electron to form an adsorbed H-atom. However, working in the blended electrolyte environment allows us to ask: is this the only contribution water makes in this reaction, or are additional water molecules involved in facilitating the reaction? Figure $2 \mathrm{C}$ illustrates the dependence of HER on both water concentration (blue) as well as water activity (red) at a constant potential versus $\mathrm{Me}_{10}-\mathrm{Fc}$. To our knowledge, there is no precedent for the reporting of the water-dependence of HER in this manner. The line fit through the blue data with $\log$ (concentration) on the x-axis, which exhibits "saturation"-type curvature, exhibits a slope of $1.74 \pm 0.13$, while the line fit to the red data with $\log$ (activity) on the $x$-axis gives a slope of $2.78 \pm 0.44$. It is therefore clear regardless of the $\mathrm{x}$-axis transformation that HER in an alkaline blended electrolyte on $\mathrm{Au}$ is dependent on water to a greater-than- ${ }^{\text {st }}$ degree. However, in analyzing the fit to the concentration-based data, one might reasonably propose mechanisms in which two distinct water molecules participate in the reaction - for instance, one as a reactant and one to coordinate the reacting molecule, or perhaps to shuttle a proton toward the electrode following a reaction. In processing the data with $\log$ (activity) on the $\mathrm{x}$-axis, it becomes apparent that any simple proposed mechanistic picture involving distinct water molecules is insufficient to explain the reaction kinetics. A 2.78-order water dependence is quite high, and without the invocation of pre-equilibrated surface intermediates (which is incompatible with the assertion of a Volmer rate-determining step), it cannot be justified by the direct participation of $\sim 2.78$ water molecules in the reaction - they would all need to participate in a single elementary step. The activity-based data, then, has opened the door of mechanistic interpretation to additional complexity, which we can here begin to further examine.

\section{The many roles of water}

Water, despite its ubiquity in electrochemistry and (electro)catalysis, is an interesting edge case when it comes to intermolecular interactions. As mentioned previously, water has a strong tendency toward self-interaction, making mixtures of water and other solvents highly nonideal. Moreover, water has several other interesting properties such as its small size, strong dipole, and Grotthuss-style diffusion mechanism for the transfer of protons and hydroxides. ${ }^{7,64,65}$ These properties are especially amplified at an electrified interface. ${ }^{56}$ As a result, there are many roles water can play in an electrocatalytic reaction. A non-exhaustive selection of such contributions is illustrated here. (Figure 3) 


\section{Roles of water...}
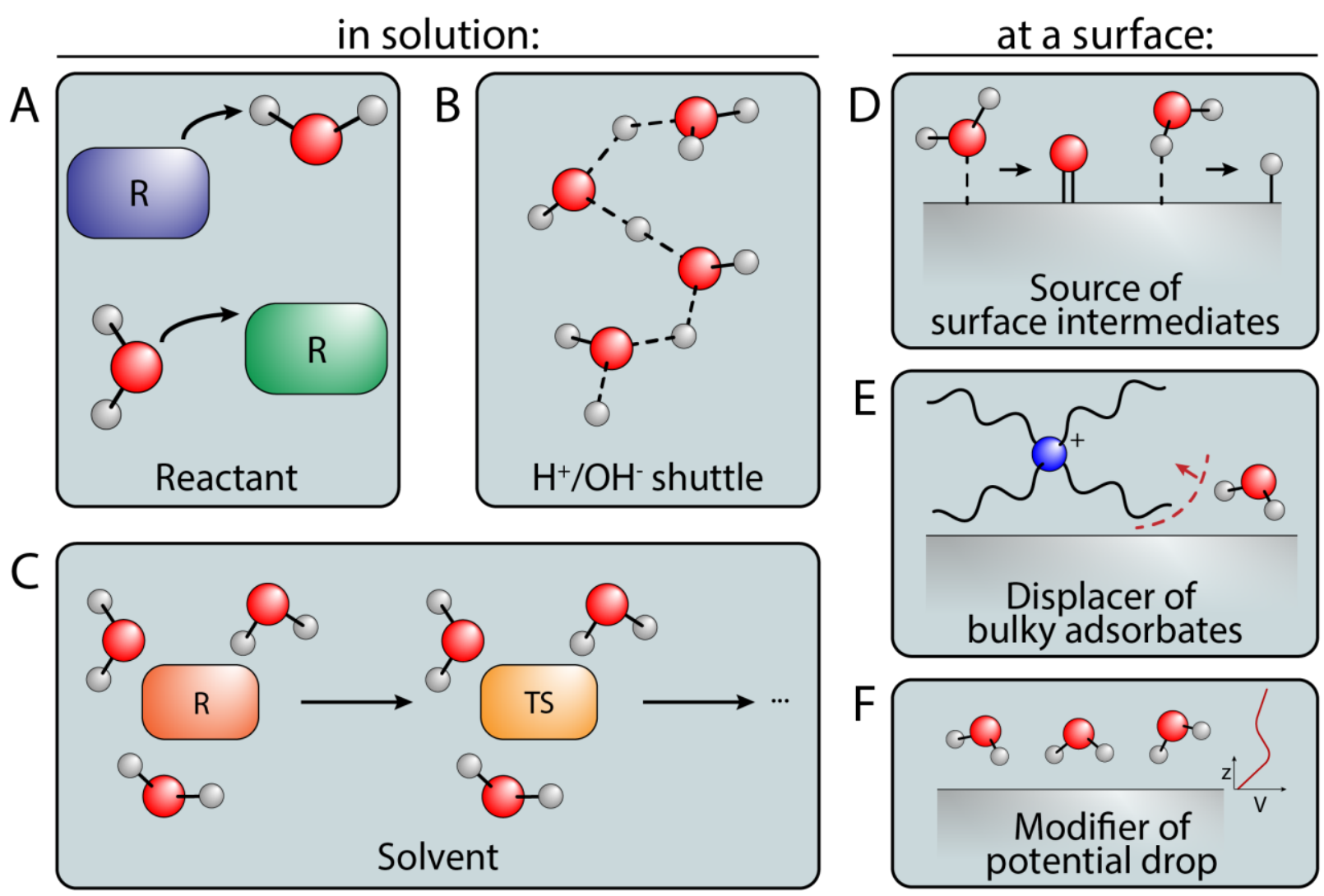

Figure 3. Schematics showing selected roles of water in an electrocatalytic reaction: A) as a reactant, B) a proton/hydroxide shuttle, C) a solvent, D) a source of surface intermediates, E) a displacer of bulky adsorbates such at the $\mathrm{TBA}^{+}$ion, and $\mathrm{F}$ ) a modifier of the interfacial potential drop. Note that roles (A), (B), and (C) can also apply at interfaces.

Beyond direct participation as a reactant in solution phase (A) and/or at a surface (D), this scheme illustrates that water can also behave in many other ways. Water can act as a buffer, shuttling protons and hydroxide ions (B); it can also modify the solvation of reacting species (C). For instance, reactive intermediates' disruption of water H-bonding networks has been implicated in observed water orders of -3 in zeolite catalysis ${ }^{66}$ Water can also displace surface adsorbates such as $\mathrm{TBA}^{+}$and acetonitrile $(\mathrm{E})^{7,67}$ - while itself blocking surface sites at the same time. In addition, we hypothesize that it can modify the shape and profile of the potential drop at an interface (F), much as ions have been shown to do in the context of aqueous electrolytes. ${ }^{68}$ On top of these effects, water can participate in specific electrostatic interactions at an interface.

When considering how to justify the 2.78-order water dependence, then, we are left with many possibilities. For instance, it would be reasonable to hypothesize that water participates both as a proton donor (D) and as a hydroxide shuttle (B) - or at least that some screening or diffusion of the resulting negative charge is required as the Volmer step proceeds. The question is, to what 
extent does hydroxide shuttling ability depend on water activity? We could equally easily postulate that outside of water's direct role as a surface reactant (D), the only other way it acts is to displace surface adsorbates such as $\mathrm{TBA}^{+}$or acetonitrile; it is also possible that the transition state during the Volmer step is more stabilized by the presence of water as a solvent $(\mathrm{C})$, to the point that $\gamma_{+}$is proportional to $a_{H 2 O^{-1.78}}$. Clearly, additional evidence is required in order to elucidate the origin of the 2.78 order dependence on water.

In addition to affecting the water order dependence, it is possible that the adsorbate displacement and potential-drop modification mechanisms illustrated in Figure $3 \mathrm{E} \& \mathrm{~F}$ contribute to the observed increase in Tafel slopes at lower electrolyte water content. Moreover, the contributions of water as a solvent and proton shuttle (Figure 3 B,C) leading to decreased Tafel slopes at higher concentrations are also in line with reports of higher concentrations of cations contributing to more facile water reduction. ${ }^{62}$ Just as cations have been implicated in stabilizing the transition state for the Volmer step in alkaline aqueous media, so too can water fill this role for itself in the absence of strongly coordinating cations.

It is worth noting at this point that there is no reason to believe the combination of all these effects should yield an integer-order dependence of a reaction rate on a reactant, especially a reactant with as many roles as water. Moreover, although it is certainly the case that reactant species activity should be a relevant descriptor for reaction rate, there is similarly no reason to expect a linear relationship between $\log$ (rate) and $\log$ (reactant activity). This is because the different roles fulfilled by the reactant of interest are liable to change across a wide concentration range. For instance, trace water has been implicated in surface accumulation even in nominally nonaqueous electrolytes; ${ }^{42}$ therefore, perhaps water's roles at a polarized interface are accentuated at low concentrations. On the other hand, maybe water only plays a traditional homogeneous solvating role for reacting species at much higher concentrations. Because the prevalence of the contributions of any arbitrary solution component can shift as one traverses the selected range of concentrations, it stands to reason that, in general, order-dependence measurements may exhibit curvature even if the direct reaction mechanism itself is not changing.

When a dependence is observed to be linear, it is worth considering what this might mean for the mechanism in question. Such an observation could arise from very consistent contributions of the reactant of interest across the activity range of interest; however, it could also arise due to fortuitous cancellations of different effects as they concurrently become more and/or less relevant to the reaction rate. To fully understand an order-dependence study in this context, it is necessary to tease apart the various roles of the reactant of interest through electrolyte and catalyst design as well as through spectroscopic and modeling approaches.

\section{Extension to other chemistries}

In order to demonstrate the broad applicability of our approach with respect to chemistry, we have also conducted water activity measurements - again in acetonitrile-based blended electrolytes for the analysis of two previously-reported oxygen-atom transfer chemistries: olefin epoxidation ${ }^{9}$ and the lactonization of cyclic ketones ${ }^{10}$ (Figure 4). Both of these chemistries have been shown to 
exhibit a nonzero rate dependence on water because water acts as the oxygen-atom source in these transformations. After measuring water activities in the exact electrolytes used for these two chemistries via headspace-gas chromatography-TCD (HS-GC-TCD) (Figure 4A,B), we shift the values on the $\mathrm{x}$-axis of the water dependence plots to reflect water activity rather than water concentration. (Figure 4C,D)
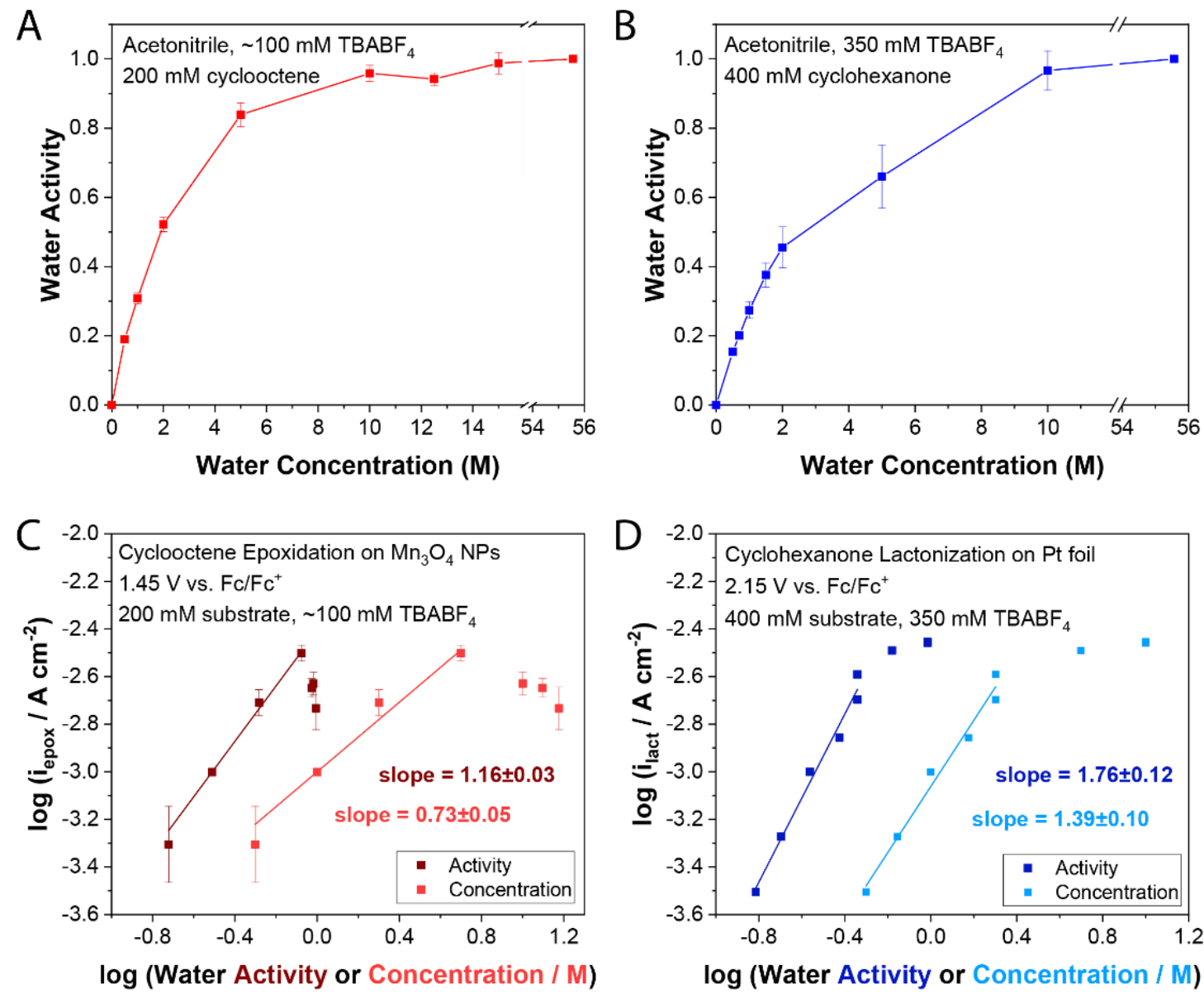

Figure 4. The effect of accounting for species activity for $\mathrm{O}$-atom transfer chemistries in blended electrolytes. A) Water activity-concentration relationship in epoxidation electrolyte, consisting of acetonitrile, $100 \mathrm{mM} \mathrm{TBABF}_{4}$, and $200 \mathrm{mM}$ cyclooctene. B) Water activity-concentration relationship in lactonization electrolyte, consisting of acetonitrile, $350 \mathrm{mM} \mathrm{TBABF} 4$, and 400 $\mathrm{mM}$ cyclohexanone. C) Water dependence data for cyclooctene epoxidation on $\mathrm{Mn}_{3} \mathrm{O}_{4}$ nanoparticles at $+1.45 \mathrm{~V}$ vs. Fc, with concentration (light red) and activity (dark red) as descriptors for water availability, as shown in Jin et al. ${ }^{9}$ D) Water dependence data for cyclohexanone lactonization on $\mathrm{Pt}$ at $+2.15 \mathrm{~V}$ vs. Fc, with concentration (light blue) and activity (dark blue) as descriptors, as shown in Maalouf et al. ${ }^{10}$ Note that in the case of (A) and (C), cyclooctene epoxidation, phase separation (seemingly of the substrate) was observed above $\sim 10$ M water. 
It can be observed that the lighter-colored data in (C) and (D) - plotted with water concentration as the operative variable - exhibit significantly different slopes when re-plotted as the darkercolored data (C) and (D) using water activity on the x-axis. These plots suggest that what was previously interpreted as "saturation" behavior - i.e., a waning dependence on water at higher concentrations - may be partially attributable to the curvature of the water activity-concentration relationship above $\sim 1 \mathrm{M}$. Some saturation behavior remains after correcting for activity coefficients of water, but as discussed previously, this is not necessarily due to a shift in the mechanism of each reaction in terms of the direct participation of water. Moreover, in the case of cyclohexanone lactonization on $\mathrm{Pt}$, this correction suggests an altogether different interpretation of the mechanism - favoring a rate expression that is closer $2^{\text {nd }}$-order with respect to water rather than $1^{\text {st }}$-order. While this conclusion can be easily justified, it would not have been evident without the application of the activity-concentration correction.

Further, it should be noted that for these chemistries which also require an organic substrate, water content can have a substantial effect on substrate activity as well. We were able to perform limited tests on the effect of water content on substrate activity, with inconclusive results. (see Supplementary Information) It stands to reason, however, that the activity of hydrophobic substrates may increase with increasing water content - this is qualitatively borne out in the observed phase separation of the epoxidation electrolyte at high water content. Because the rate of cyclooctene epoxidation was shown to be proportional to $c_{\text {cyclooctene }}{ }^{0.7}$, it is possible that the two order dependence measurements are interdependent on one another. In cases such as this, it is advisable to carefully measure the effects of one species' activity on another species' activity, and to design experiments such that one species' activity is held constant while the other is varied. This may require the formulation of electrolytes that contain different concentrations of a component whose activity is being held constant, which may seem counter-intuitive. For example, when conducting a water dependence experiment for cyclooctene epoxidation, it may be necessary to decrease the volume of cyclooctene added to the electrolyte as the water content increases, so that the activity of cyclooctene is the same at all tested conditions.

\section{Toward a molecular picture of species activity}

A desire for molecular understanding of how the water activity-concentration relationship arises in the blended acetonitrile-water system drove us to investigate this system using molecular dynamics simulations. (Figure 5) Molecular dynamics simulation systems composed of acetonitrile molecules,${ }^{69}$ tetrabutylammonium cations,${ }^{70}$ and tetrafluoroborate anions (force field parameters taken from Liu et al. ${ }^{71}$ ), and a variable number of water molecules (using the TIP3P force field with SHAKE constraints) ${ }^{72}$ were constructed using the Packmol software. ${ }^{73}$ (see Methods) It should be noted that the force field used in these simulations has not been rigorously validated in the context of the blended electrolyte; as such, the simulation results should not be taken quantitatively, especially those at very low concentrations of water. 

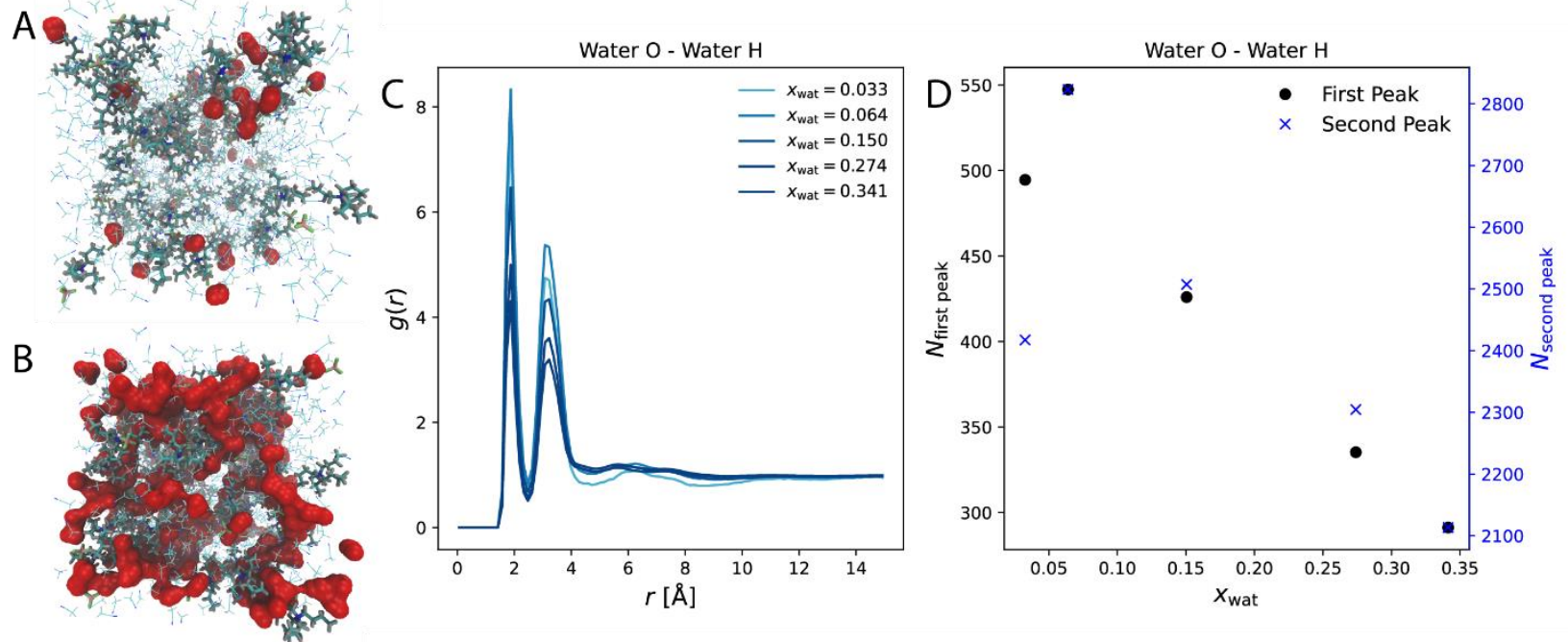

Figure 5. Results of molecular dynamics simulations of the acetonitrile-water blended electrolyte with $0.8 \mathrm{M} \mathrm{TBABF}_{4}$. A) Snapshot of $0.5 \mathrm{M}$ water condition; B) snapshot of $6.6 \mathrm{M}$ water condition; C) radial distribution function showing relative abundance of water $\mathrm{H}$-atoms as a function of radius from a central water O-atom; D) plot showing integrated areas of the first and second water peaks from (C) for each composition, versus electrolyte water mole fraction. In (A) and $(\mathrm{B})$, water is shown as a red surface; $\mathrm{TBA}^{+}$and $\mathrm{BF}_{4}^{-}$are shown in the licorice representation with carbon light blue, nitrogen blue, hydrogen gray, boron pink, and fluorine lime green; and acetonitrile in lines representation with the same color scheme.

Figure 5A \& B are snapshots of the electrolyte box at low $(0.5 \mathrm{M})$ and high $(6.6 \mathrm{M})$ concentrations of water, respectively. Similar to previously reported simulations, ${ }^{7}$ we observe relatively isolated water molecules at low concentrations and clusters or "nanodomains" at higher concentrations. The hydrophobic $\mathrm{TBA}^{+}$ions appear to carve out pockets where water is less concentrated, while water in the "nanodomains" becomes energetically similar to bulk water.

It is fairly straightforward to interpret the molecular pictures shown here to justify the concavedown trend in the activity curves (Figure 1, Figure 2A) in the dilute-water limit. At the lowest concentrations of water (Figure 5A), most water molecules are completely surrounded by acetonitrile and $\mathrm{TBABF}_{4}$ - perhaps as little as $10-20 \%$ of water molecules are H-bonded to other water molecules. At this condition the chemical potential of any single water molecule is quite high, as it is engaging in less favorable interactions than it would be in a pure water phase. As water is added into the system and we approach near-saturation conditions (Figure 5B), "wires" and even "domains" of water form in which water molecules are clustered with themselves. Under these conditions, the water molecules engage in more stabilizing interactions, and the chemical potential of any one water molecule is less than that of a water molecule in the dilute-water case. That is, the measured "energy per additional molecule" decreases as the concentration of water increases. This corresponds to a decrease in activity coefficient with increasing water concentration, and is borne out as a concave-down trend in the activity-concentration relationship. Notably, we can see from the radial distribution function showing water $\mathrm{O}$ - water $\mathrm{H}$ distances in 
Figure 5C, as well as the plot of the $\mathrm{g}(\mathrm{r})$ peak area values in Figure 5D, that water does have a propensity to cluster with other water molecules at all concentrations. The absolute abundance of water clusters increases with increasing concentration, as it is of course entropically disfavored for all water in the low-concentration limit to remain bound to itself. However, there is an enthalpic driving force that encourages excess water coordination, especially at the conditions of highest chemical potential per molecule of water.

A question that remains through this analysis, however, is whether certain sub-populations of water - such as dimers or tri+-mers - are more reactive than other sub-populations - for example, lone water molecules. While the probability of finding these populations gradually changes with water activity (Figure 5D), it is perhaps remarkable that despite all such reasoning, water activity remains the descriptor in the system that tends to linearize observed experimental trends.

While these results should be considered only preliminary, they are interesting enough to warrant validation in an experimental setup - which we strongly advocate. In the future, it would be interesting to take the further step of calculating activities directly from simulations as part of this validation, as well as to spectroscopically probe the structure of water in bulk blended electrolytes. In addition, due to the catalytic relevance of the surface, it is worth assessing the interfacial structure of water in the blended electrolyte - ideally under polarization - through both simulation as well as spectroscopy.

\section{Discussion}

In this work, we emphasize the importance of directly measuring the thermodynamic activity of species relevant to electrocatalytic reactions in order to properly interpret electrochemical kinetic data. Headspace gas chromatography techniques have been used toward this end. Upon applying a correction to account for the species activity coefficient in the solvent of interest, water dependence data for HER as well as previously studied O-atom transfer chemistries yields water orders that were previously obscured by changes in activity coefficient. However, these dependences do not necessarily exhibit linear slopes, nor slopes indicative of integer-order dependence, because of the many roles that water can play in the electrochemical system. Water mixtures are particularly prone to being affected by nonidealities, although any mixed system consisting of components that exhibit intermolecular interactions that strongly differ from selfinteractions will also yield substantial nonidealities. Finally, we also speculate at the molecular origins of the water activity-concentration relationship in the blended electrolyte, for which validation of molecular simulations with experimental data is required. Principles discussed in this work are applicable to the deconvolution of system parameters in the future study of electrochemical active species dependence as well as solvent effects. Further, the relevance of these conclusions also extends to environments similar to blended electrolytes where local solvation deviates from that of a pure solvent, such as in zeolites and metal-organic frameworks. 


\section{Methods}

Headspace-Gas Chromatography-TCD measurements for activity quantification

Each tested electrolyte composition was prepared in a $20-\mathrm{mL}$ glass headspace vial under unaltered lab atmosphere, then capped and allowed to equilibrate at room temperature $\left(\sim 20-23{ }^{\circ} \mathrm{C}\right)$ for at least 12 hours. An Agilent automated headspace sampler then sampled from the electrolyte headspace, sending each sample through a DB 624-UI gas chromatography column that led to a thermal conductivity detector (TCD). The ideal gas law was assumed to hold for these headspace samples - a generally good assumption for air at STP. A pure water sample was taken in-between consecutive electrolyte samples. Using a Raoult's law referencing scheme in which the activity of pure water was defined as being equal to 1 , the activity of water in each electrolyte was calculated as:

$$
a_{H 2 O, \text { sample }}=\frac{A_{H 2 O, \text { sample }}}{A_{H 2 O, \text { pure }}}
$$

Triplicate measurements were conducted in this way to generate Figure 2A.

\section{HER experiments: Au disk in RDE}

The Au disk used in experiments was prepared ${ }^{74}$ by consecutive polishes with $0.3 \mu \mathrm{m}$ alumina on a vinyl pad (30 sec $-2 \mathrm{~min}$ ) and $0.05 \mu \mathrm{m}$ alumina MicroPolish on a microfiber pad ( $2 \mathrm{~min})$, then sonicated twice in fresh 50:50 Milli-Q:acetone by volume ( 2 min each). The disk was then electro-polished by inserting it into a rotator and cycling $200 \mathrm{x}$ between 0 and $+1.800 \mathrm{~V}$ vs. a Pt wire counter electrode at a rate of $1 \mathrm{~V} / \mathrm{sec}$ in an electrolyte of $0.1 \mathrm{M} \mathrm{H}_{2} \mathrm{SO}_{4}$. After a double-rinse with Milli-Q and drying with a Kimwipe, the electrode was ready for use. The RDE was inserted into a custom $\mathrm{H}$-cell containing the working electrolyte, leak-free $\mathrm{Ag} / \mathrm{AgCl}$ pseudo-reference electrode, as well as Pt wire counter electrode. The working compartment was sealed using a Pine Gas-Purged Bearing into which $20 \mathrm{sccm}$ Ar gas was flowed to blanket the electrolyte and maintain an inert atmosphere. After reacting away the dissolved oxygen gas in solution, LSVs performed at a sweep rate of $-2 \mathrm{mV} / \mathrm{sec}$ yielded the Tafel plots shown in Figure 2B. A cut through at a constant potential versus $\mathrm{Me}_{10}-\mathrm{Fc}$ on Figure $2 \mathrm{~B}$, combined with the activity concentration relationship in Figure 2A, yielded the water dependence shown in Figure 2C.

\section{Molecular dynamics simulations}

The simulated MD systems were initially relaxed using conjugate gradient minimization, and then the system volume was relaxed over the course of a 1 ns NpT ensemble simulation using the NoseHoover thermostat and barostat, with an integration timestep of $1.0 \mathrm{fs}$. Upon volume relaxation, systems were prepared at the equilibrated densities, followed by a production NVT simulation of $1 \mathrm{~ns}$ using the Nose-Hoover thermostat, with a timestep of 1.5 fs. Densities of simulated electrolytes were found to be within $10 \%$ of experimental values. 


\section{Acknowledgements}

The authors would like to thank Thejas Wesley for insightful contributions on nonideal rate laws in the transition state theory framework. We also appreciate feedback from James Mayer, Morris Bullock, Catherine Wise, and Rishi Agarwal on the measurement of RHE in nonaqueous electrolytes. In addition, we are grateful to Zachary Schiffer, Nikifar Lazouski, Nathan Corbin, Joy Zeng, Joseph Maalouf, Katherine Steinberg, Simar Mattewal, Fang-Yu Kuo, Sayandeep Biswas, Kyoungsuk Jin, Deng-Tao Yang, and Hee Jo Song for ongoing discussions and feedback on this work. This research was supported as part of the Center for Molecular Electrocatalysis, an Energy Frontier Research Center funded by the U.S. Department of Energy, Office of Science, Office of Basic Energy Sciences.

\section{Author Information}

\section{ORCID}

Kindle Williams: 0000-0001-9640-7849

Aditya Limaye: 0000-0003-0639-4154

Minju Chung: 0000-0003-4359-7508

Karthish Manthiram: 0000-0001-9260-3391

\section{Author Contributions}

Conceptualization: K.S.W. and K.M.; experiments: K.S.W.; MD simulations: A.M.L.; reproduction: M.C. and K.S.W.; writing (original draft, all except simulation portion): K.S.W.; writing (original draft, simulation portion): A.M.L. and K.S.W.; writing (review and editing): K.S.W., T.A.W., M.C., A.M.L., and K.M.; supervision: K.M.

\section{References}

(1) Schiffer, Z. J.; Manthiram, K. Electrification and Decarbonization of the Chemical Industry. Joule 2017, 1 (1), 10-14.

(2) Sasaki, K.; Kashimura, T.; Ohura, M.; Ohsaki, Y.; Ohta, N. Solvent Effect in the Electrochemical Reduction of P-Quinones in Several Aprotic Solvents. J. Electrochem. Soc. 1990, 137 (8), 2437-2443.

(3) Park, J. W.; Yamauchi, K.; Takashima, E.; Tachikawa, N.; Ueno, K.; Dokko, K.; Watanabe, M. Solvent Effect of Room Temperature Ionic Liquids on Electrochemical Reactions in Lithium-Sulfur Batteries. J. Phys. Chem. C 2013, 117 (9), 4431-4440.

(4) König, M.; Vaes, J.; Klemm, E.; Pant, D. Solvents and Supporting Electrolytes in the Electrocatalytic Reduction of CO2. iScience 2019, 19, 135-160. 
(5) Barrette, W. C.; Sawyer, D. T. Determination of Dissolved Hydrogen and Effects of Media and Electrode Materials on the Electrochemical Oxidation of Molecular Hydrogen. Anal. Chem. 1984, 56 (4), 653-657.

(6) Ringe, S.; Clark, E. L.; Resasco, J.; Walton, A.; Seger, B.; Bell, A. T.; Chan, K. Understanding Cation Effects in Electrochemical CO2 Reduction. Energy Environ. Sci. 2019, 12 (10), 3001-3014.

(7) Dubouis, N.; Serva, A.; Berthin, R.; Jeanmairet, G.; Porcheron, B.; Salager, E.; Salanne, M.; Grimaud, A. Tuning Water Reduction through Controlled Nanoconfinement within an Organic Liquid Matrix. Nat. Catal. 2020, 3, 656-663.

(8) Monteiro, M. C. O.; Goyal, A.; Moerland, P.; Koper, M. T. M. Understanding Cation Trends for Hydrogen Evolution on Platinum and Gold Electrodes in Alkaline Media. ACS Catal. 2021, 14328-14335.

(9) Jin, K.; Maalouf, J.; Lazouski, N.; Corbin, N.; Yang, D.; Manthiram, K. Epoxidation of Cyclooctene Using Water as Oxygen-Atom Source at Manganese Oxide Electrocatalysts. J. Am. Chem. Soc. 2019, 141 (15), 6413-6418.

(10) Maalouf, J.; Jin, K.; Yang, D.; Limaye, A.; Manthiram, K. Kinetic Analysis of Electrochemical Lactonization of Ketones Using Water as the Oxygen Atom Source. ACS Catal.

(11) Hatay, I.; Su, B.; Li, F.; Partovi-Nia, R.; Vrubel, H.; Hu, X.; Ersoz, M.; Girault, H. H. Hydrogen Evolution at Liquid-Liquid Interfaces. Angew. Chemie - Int. Ed. 2009, 48 (28), 5139-5142.

(12) Terry Weatherly, C. K.; Ren, H.; Edwards, M. A.; Wang, L.; White, H. S. Coupled Electronand Phase-Transfer Reactions at a Three-Phase Interface. J. Am. Chem. Soc. 2019, 141 (45), 18091-18098.

(13) Dong, F.; Chen, H.; Malapit, C. A.; Prater, M. B.; Li, M.; Yuan, M.; Lim, K.; Minteer, S. D. Biphasic Bioelectrocatalytic Synthesis of Chiral $\beta$-Hydroxy Nitriles. J. Am. Chem. Soc. 2020, 142 (18), 8374-8382.

(14) Noël, T.; Cao, Y.; Laudadio, G. The Fundamentals behind the Use of Flow Reactors in Electrochemistry. Acc. Chem. Res. 2019, 52 (10), 2858-2869.

(15) Chen, H.; Cong, G.; Lu, Y.-C. Recent Progress in Organic Redox Flow Batteries: Active Materials, Electrolytes and Membranes. J. Energy Chem. 2018, 27 (5), 1304-1325.

(16) Stace, A. J. Preferential Solvation of Hydrogen Ions in Mixed Water-Amine Ion Clusters. J. Am. Chem. Soc. 1984, 106 (8), 2306-2315.

(17) Mestdagh, J. M.; Binet, A.; Sublemontier, O. Solvation Shells of the Proton Surrounded by Acetonitrile, Ethanol, and Water Molecules. J. Phys. Chem. 1989, 93 (26), 8300-8303.

(18) Jamroz, D.; Stangret, J.; Lindgren, J.; Jamroz, D.; Stangret, J. An Infrared Spectroscopic Study of the Preferential Solvation in Water-Acetonitrile Mixtures. J. Am. Chem. Soc. 1993, 115 (14), 6165-6168. 
(19) Kovacs, H.; Laaksonen, A. Molecular Dynamics Simulation and NMR Study of WaterAcetonitrile Mixtures. J. Am. Chem. Soc. 1991, 113 (15), 5596-5605.

(20) Guo, J.-H.; Luo, Y.; Augustsson, A.; Kashtanov, S.; Rubensson, J.-E. E.; Shuh, D. K. D.; Agren, H.; Nordgren, J.; Ågren, H.; Nordgren, J. Molecular Structure of Alcohol-Water Mixtures. Phys. Rev. Lett. 2003, 91 (15), 1-4.

(21) Kashtanov, S.; Augustson, A.; Rubensson, J.-E.; Nordgren, J.; Ågren, H.; Guo, J.-H.; Luo, Y. Chemical and Electronic Structures of Liquid Methanol from X-Ray Emission Spectroscopy and Density Functional Theory. Phys. Rev. B 2005, 71, 104205.

(22) Verma, A.; Stoppelman, J. P.; McDaniel, J. G. Tuning Water Networks via Ionic Liquid/Water Mixtures. Int. J. Mol. Sci. 2020, 21 (2).

(23) Suo, L.; Borodin, O.; Gao, T.; Olguin, M.; Ho, J.; Fan, X.; Luo, C.; Wang, C.; Xu, K. "Water-in-Salt" Electrolyte Enables High-Voltage Aqueous Lithium-Ion Chemistries. Science (80-. ). 2015, 350 (6263), 938-943.

(24) Shuai, L.; Luterbacher, J. Organic Solvent Effects in Biomass Conversion Reactions. ChemSusChem 2016, 9 (2), 133-155.

(25) Mellmer, M. A.; Sanpitakseree, C.; Demir, B.; Bai, P.; Ma, K.; Neurock, M.; Dumesic, J. A. Solvent-Enabled Control of Reactivity for Liquid-Phase Reactions of Biomass-Derived Compounds. Nat. Catal. 2018, 1 (3), 199-207.

(26) Chew, A. K.; Walker, T. W.; Shen, Z.; Demir, B.; Witteman, L.; Euclide, J.; Huber, G. W.; Dumesic, J. A.; Van Lehn, R. C. Effect of Mixed-Solvent Environments on the Selectivity of Acid-Catalyzed Dehydration Reactions. ACS Catal. 2020, 10 (3), 1679-1691.

(27) Angamuthu, R.; Bouwman, E. Reduction of Protons Assisted by a Hexanuclear Nickel Thiolate Metallacrown: Protonation and Electrocatalytic Dihydrogen Evolution. Phys. Chem. Chem. Phys. 2009, 11 (27), 5578-5583.

(28) Lee, C. H.; Dogutan, D. K.; Nocera, D. G. Hydrogen Generation by Hangman Metalloporphyrins. J. Am. Chem. Soc. 2011, 133 (23), 8775-8777.

(29) Garcia-Araez, N.; Climent, V.; Feliu, J. Potential-Dependent Water Orientation on Pt(111), $\operatorname{Pt}(100)$, and $\operatorname{Pt}(110)$, as Inferred from Laser-Pulsed Experiments. Electrostatic and Chemical Effects. J. Phys. Chem. C 2009, 113 (21), 9290-9304.

(30) Roman, T.; Groß, A. Structure of Water Layers on Hydrogen-Covered Pt Electrodes. Catal. Today 2013, 202 (1), 183-190.

(31) Lam, Y. C.; Soudackov, A. V.; Hammes-Schiffer, S. Kinetics of Proton Discharge on Metal Electrodes: Effects of Vibrational Nonadiabaticity and Solvent Dynamics. J. Phys. Chem. Lett. 2019, 10 (18), 5312-5317.

(32) Ledezma-Yanez, I.; Wallace, W. D. Z.; Sebastián-Pascual, P.; Climent, V.; Feliu, J. M.; Koper, M. T. M. Interfacial Water Reorganization as a PH-Dependent Descriptor of the Hydrogen Evolution Rate on Platinum Electrodes. Nat. Energy 2017, 2 (4), 1-7.

Dubouis, N.; Serva, A.; Salager, E.; Deschamps, M.; Salanne, M.; Grimaud, A. The Fate of 
Water at the Electrochemical Interfaces: Electrochemical Behavior of Free Water Versus Coordinating Water. J. Phys. Chem. Lett. 2018, 9 (23), 6683-6688.

(34) Yang, X.; Nash, J.; Oliveira, N.; Yan, Y.; Xu, B. Understanding the PH Dependence of Underpotential Deposited Hydrogen on Platinum. Angew. Chemie - Int. Ed. 2019, 58 (49), 17718-17723.

(35) Liu, E.; Li, J.; Jiao, L.; Doan, H. T. T.; Liu, Z.; Zhao, Z.; Huang, Y.; Abraham, K. M.; Mukerjee, S.; Jia, Q. Unifying the Hydrogen Evolution and Oxidation Reactions Kinetics in Base by Identifying the Catalytic Roles of Hydroxyl-Water-Cation Adducts. J. Am. Chem. Soc. 2019, 141 (7), 3232-3239.

(36) Liu, E.; Jiao, L.; Li, J.; Stracensky, T.; Sun, Q.; Mukerjee, S.; Jia, Q. Interfacial Water Shuffling the Intermediates of Hydrogen Oxidation and Evolution Reactions in Aqueous Media. Energy Environ. Sci. 2020, 13 (9), 3064-3074.

(37) Rebollar, L.; Intikhab, S.; Oliveira, N. J.; Yan, Y.; Xu, B.; McCrum, I. T.; Snyder, J. D.; Tang, M. H. "Beyond Adsorption" Descriptors in Hydrogen Electrocatalysis. ACS Catal. 2020, 10 (24), 14747-14762.

(38) Cheng, T.; Wang, L.; Merinov, B. V.; Goddard, W. A. Explanation of Dramatic PHDependence of Hydrogen Binding on Noble Metal Electrode: Greatly Weakened Water Adsorption at High PH. J. Am. Chem. Soc. 2018, 140 (25), 7787-7790.

(39) Sarabia, F. J.; Sebastián-Pascual, P.; Koper, M. T. M.; Climent, V.; Feliu, J. M. Effect of the Interfacial Water Structure on the Hydrogen Evolution Reaction on $\mathrm{Pt}(111)$ Modified with Different Nickel Hydroxide Coverages in Alkaline Media. ACS Appl. Mater. Interfaces 2019, $11(1), 613-623$.

(40) Ranninger, J.; Mayrhofer, K. J. J.; Berkes, B. B. The Crucial Role of Water in the Stability and Electrocatalytic Activity of Pt Electrodes. J. Phys. Chem. C 2021, 125 (24), 1325413263.

(41) Ledezma-Yanez, I.; Díaz-Morales, O.; Figueiredo, M. C.; Koper, M. T. M. Hydrogen Oxidation and Hydrogen Evolution on a Platinum Electrode in Acetonitrile. ChemElectroChem 2015, 2 (10), 1612-1622.

(42) Ledezma-Yanez, I.; Koper, M. T. M. Influence of Water on the Hydrogen Evolution Reaction on a Gold Electrode in Acetonitrile Solution. J. Electroanal. Chem. 2017, 793, $18-24$.

(43) Lanning, J. A.; Chambers, J. Q. Voltammetric Study of the Hydrogen Ion/Hydrogen Couple in Acetonitrile/Water Mixtures. Anal. Chem. 1973, 45 (7), 1010-1016.

(44) Suárez-Herrera, M. F.; Costa-Figueiredo, M.; Feliu, J. M. Voltammetry of Basal Plane Platinum Electrodes in Acetonitrile Electrolytes: Effect of the Presence of Water. Langmuir 2012, 28 (11), 5286-5294.

(45) Castelli, I. E.; Zorko, M.; Østergaard, T. M.; Martins, P. F. B. D.; Lopes, P. P.; Antonopoulos, B. K.; Maglia, F.; Markovic, N. M.; Strmcnik, D.; Rossmeisl, J. The Role of an Interface in Stabilizing Reaction Intermediates for Hydrogen Evolution in Aprotic Electrolytes. Chem. Sci. 2020. 
(46) Roberts, J. A. S. S.; Bullock, R. M. Direct Determination of Equilibrium Potentials for Hydrogen Oxidation/Production by Open Circuit Potential Measurements in Acetonitrile. Inorg. Chem. 2013, 52 (7), 3823-3835.

(47) Bautista-Martinez, J. A.; Tang, L.; Zeller, R.; Angell, C. A.; Friesen, C. Hydrogen Redox in Protic Ionic Liquids and a Direct Measurement of Proton Thermodynamics. J. Phys. Chem. C 2009, 113, 12586-12593.

(48) O'Reilly, N. J.; Magner, E. Electrochemistry of Cytochrome c in Aqueous and Mixed Solvent Solutions: Thermodynamics, Kinetics, and the Effect of Solvent Dielectric Constant. Langmuir 2005, 21 (3), 1009-1014.

(49) Matsubara, Y. Unified Benchmarking of Electrocatalysts in Noninnocent Second Coordination Spheres for CO2 Reduction. ACS Energy Lett. 2019, 4 (8), 1999-2004.

(50) Briega-Martos, V.; Costa-Figueiredo, M.; Orts, J. M.; Rodes, A.; Koper, M. T. M.; Herrero, E.; Feliu, J. M. Acetonitrile Adsorption on Pt Single-Crystal Electrodes and Its Effect on Oxygen Reduction Reaction in Acidic and Alkaline Aqueous Solutions. J. Phys. Chem. C 2019, 123 (4), 2300-2313.

(51) Briega-Martos, V.; Solla-Gullón, J.; Koper, M. T. M.; Herrero, E.; Feliu, J. M. Electrocatalytic Enhancement of Formic Acid Oxidation Reaction by Acetonitrile on WellDefined Platinum Surfaces. Electrochim. Acta 2019, 295, 835-845.

(52) D'Elia, L. F.; Ortiz, R. L. Electrochemical Oxidation of Toluene on Glassy Carbon in Organic-Aqueous Acid Solution. J. Electrochem. Soc. 2006, 153 (12), D187.

(53) Madon, R. J.; Iglesia, E. Catalytic Reaction Rates in Thermodynamically Non-Ideal Systems. J. Mol. Catal. A Chem. 2000, 163 (1-2), 189-204.

(54) Treiner, C.; Tzias, P.; Chemla, M. Solvation of Tetrabutylammonium Bromide in Water Acetonitrile Mixtures at 298.15 K from Vapour Pressure Measurements of Dilute Solutions. J. Chem. Soc. Faraday Trans. 1 1976, 72, 2007-2015.

(55) Sirotkin, V. A.; Solomonov, B. N.; Faizullin, D. A.; Fedotov, V. D. IR Spectroscopic Study of the State of Water in Dioxane and Acetonitrile: Relationship with the Thermodynamic Activity of Water at 278-318 K. J. Struct. Chem. 2000, 41 (6), 997-1003.

(56) Gonella, G.; Backus, E. H. G.; Nagata, Y.; Bonthuis, D. J.; Loche, P.; Schlaich, A.; Netz, R. R.; Kühnle, A.; McCrum, I. T.; Koper, M. T. M.; Wolf, M.; Winter, B.; Meijer, G.; Campen, R. K.; Bonn, M. Water at Charged Interfaces. Nat. Rev. Chem. 2021, 5 (7), 466485 .

(57) Noviandri, I.; Brown, K. N.; Fleming, D. S.; Gulyas, P. T.; Lay, P. A.; Masters, A. F.; Phillips, L. The Decamethylferrocenium/Decamethylferrocene Redox Couple: A Superior Redox Standard to the Ferrocenium/Ferrocene Redox Couple for Studying Solvent Effects on the Thermodynamics of Electron Transfer. J. Phys. Chem. B 1999, 103 (32), 6713-6722.

(58) Agarwal, R. G.; Coste, S. C.; Groff, B. D.; Heuer, A. M.; Noh, H.; Parada, G. A.; Wise, C. F.; Nichols, E. M.; Warren, J. J.; Mayer, J. M. Free Energies of Proton-Coupled Electron Transfer Reagents and Their Applications. Chem. Rev. 2021. 
(59) Wise, C. F.; Agarwal, R. G.; Mayer, J. M. Determining Proton-Coupled Standard Potentials and X-H Bond Dissociation Free Energies in Nonaqueous Solvents Using Open-Circuit Potential Measurements. J. Am. Chem. Soc. 2020, 142 (24), 10681-10691.

(60) Shinagawa, T.; Garcia-Esparza, A. T.; Takanabe, K. Insight on Tafel Slopes from a Microkinetic Analysis of Aqueous Electrocatalysis for Energy Conversion. Sci. Rep. 2015, 5 (September), 1-21.

(61) Kahyarian, A.; Brown, B.; Nesic, S. Mechanism of the Hydrogen Evolution Reaction in Mildly Acidic Environments on Gold. J. Electrochem. Soc. 2017, 164 (6), H365-H374.

(62) Goyal, A.; Koper, M. T. M. Understanding the Role of Mass Transport in Tuning the Hydrogen Evolution Kinetics on Gold in Alkaline Media. J. Chem. Phys. 2021, 155 (13).

(63) Limaye, A. M.; Zeng, J. S.; Willard, A. P.; Manthiram, K. Bayesian Data Analysis Reveals No Preference for Cardinal Tafel Slopes in CO2 Reduction Electrocatalysis. Nat. Commun. 2021, 12 (1), 1-10.

(64) de Grotthuß, C. J. D. T. Sur La Décomposition de l'eau et Des Corps Qu'elle Tient En Dissolution à l'aide de l'électricité Galvanique. Ann. di Chim. (Rome, Italy) 1805, 58, 5473.

(65) Rasaiah, J. C.; Garde, S.; Hummer, G. Water in Nonpolar Confinement: From Nanotubes to Proteins and Beyond. Annu. Rev. Phys. Chem. 2008, 59 (1), 713-740.

(66) Bates, J. S.; Bukowski, B. C.; Greeley, J.; Gounder, R. Structure and Solvation of Confined Water and Water-Ethanol Clusters within Microporous Brønsted Acids and Their Effects on Ethanol Dehydration Catalysis. Chem. Sci. 2020, 11 (27), 7102-7122.

(67) Rudnev, A. V.; Molodkina, E. B.; Danilov, A. I.; Polukarov, Y. M.; Berna, A.; Feliu, J. M. Adsorption Behavior of Acetonitrile on Platinum and Gold Electrodes of Various Structures in Solution of 0.5 M H2SO4. Electrochim. Acta 2009, 54 (14), 3692-3699.

(68) Hussain, G.; Pérez-Martínez, L.; Le, J. B.; Papasizza, M.; Cabello, G.; Cheng, J.; Cuesta, A. How Cations Determine the Interfacial Potential Profile: Relevance for the CO2 Reduction Reaction. Electrochim. Acta 2019, 327.

(69) Wu, X.; Liu, Z.; Huang, S.; Wang, W. Molecular Dynamics Simulation of RoomTemperature Ionic Liquid Mixture of [Bmim][BF4] and Acetonitrile by a Refined Force Field. Phys. Chem. Chem. Phys. 2005, 7 (14), 2771-2779.

(70) Bhowmik, D.; Malikova, N.; Mériguet, G.; Bernard, O.; Teixeira, J.; Turq, P. Aqueous Solutions of Tetraalkylammonium Halides: Ion Hydration, Dynamics and Ion-Ion Interactions in Light of Steric Effects. Phys. Chem. Chem. Phys. 2014, 16 (26), 1344713457.

(71) Liu, Z.; Huang, S.; Wang, W. A Refined Force Field for Molecular Simulation of Imidazolium-Based Ionic Liquids. J. Phys. Chem. B 2004, 108 (34), 12978-12989.

(72) Jorgensen, W. L.; Chandrasekhar, J.; Madura, J. D.; Impey, R. W.; Klein, M. L. Comparison of Simple Potential Functions for Simulating Liquid Water. J. Chem. Phys. 1983, 79 (2), 926-935. 
(73) Martínez, L.; Andrade, R.; Birgin, E. G.; Martínez, J. M. PACKMOL: A Package for Building Initial Configurations for Molecular Dynamics Simulations. J. Comput. Chem. 2009, 30 (13), 2157-2164.

(74) Goyal, A.; Marcandalli, G.; Mints, V. A.; Koper, M. T. M. Competition between CO2 Reduction and Hydrogen Evolution on a Gold Electrode under Well-Defined Mass Transport Conditions. J. Am. Chem. Soc. 2020, 142 (9), 4154-4161. 